\title{
¿CÓMO AFECTA LA CRISIS ECONÓMICA AL CONTEXTO DE LA PROSTITUCIÓN DE CALLE? ${ }^{1}$
}

\author{
How does the economic crisis affect the context of street prostitution? \\ CARMEn MENESES FALCÓN² y LAURa GuindeO AGUERRI ${ }^{3}$
}

\section{Resumen}

Este estudio tiene la finalidad de describir las repercusiones que la crisis económica ha supuesto en las poblaciones más vulnerables, en concreto en las mujeres que ejercen la prostitución en la calle, así como en las entidades que les prestan apoyo. Para desarrollar los objetivos se ha realizado trabajo etnográfico (observación participante y entrevistas) en una zona de prostitución de calle de la ciudad de Madrid. Así mismo se ha realizado entrevistas semiestructuradas a las entidades principales que trabajan con estas mujeres que ejerce la prostitución en la misma ciudad para poder triangular la información y complementarla. Entre las consecuencias destacan una reducción de los clientes y por tanto de los ingresos, con más largas horas de presencia en la calle, aceptando servicios o prácticas de riesgo a cambio de un incremento económico y la inaccesibilidad a los servicios sanitarios básicos. Los resultados nos llevan a concluir que la reducción de asistencia social y sanitaria en los colectivos más vulnerables conlleva la transformación de los mismos en grupos en exclusión social, así como posibles repercusiones en salud pública al no poder detectar y prevenir muchas de las infecciones que puedan producirse.

Palabras clave: Prostitución, exclusión social.

\begin{abstract}
This study aims to describe the impact of the economic crisis on the most vulnerable populations, particularly female street prostitutes and the institutions that support them. To obtain these goals, the study was done through fieldwork (participant observation and interviews) in a characteristic area of prostitution located in Madrid. The study was also conducted through semi-structured interviews with key organizations that work with these women engaged in prostitution in the same city, to triangulate the information to complement the study. Principal consequences include: reduced customers and therefore less revenue, longer hours on the streets, acceptance of unsafe practices to make more money, and inaccessibility of basic healthcare services. These results lead us to conclude that the reduction of health and social care in the most vulnerable population transforms them into groups of social exclusion, which may affect also the public health system, by failing to detect and prevent many possible derivate infections.
\end{abstract}

Keywords: Prostitution, social exclusion.

Recibido: 16/09/2015

Aceptado: 16/11/2015

Publicado: 03/12/2015

1. Este proyecto se ha realizado en el marco del Proyecto Nacional de I+D+I con referencia CSO201455209-P, «Visibilizando a las víctimas de trata: actuando contra la trata de mujeres con fines de explotación sexual», y ha sido aprobado por el Comité de Ética de la Universidad Pontificia Comillas.

2. Departamento de Sociología y Trabajo social. Universidad Pontificia Comillas. c/ Universidad Comillas, 3. 28049. Telf.917343950. email: cmeneses@comillas.edu

3. Instituto de Innovación Social de la Universidad de ESADE 


\section{Introducción}

La prostitución es una actividad de baja cualificación, predominantemente femenina, y en las que las mujeres pueden ganar más dinero que los hombres en su mismo nivel (Edlund y Korn, 2002). La prostitución puede ofrecer mucho dinero en pocas horas y por eso se ha asociado con una actividad de «dinero rápido» (Willman, 2010). Los riesgos de tipo físico, psicológico y social que conlleva, hace que no sea la más deseada para las mujeres entre sus alternativas de sustento (Meneses, 2007). El alto nivel de ingresos económicos, el grado de autonomía y capacidad de decisión, cuando la actividad es voluntaria, llevan a muchas mujeres a tenerla en cuenta como una importante opción para su subsistencia. Muchos estudios han puesto de manifiesto que la principal razón para ejercer la prostitución es la necesidad económica y de supervivencia, especialmente entre las capas populares (Vanwessenbeeck, 2001; Meneses et al, 2003; Monroe, 2005), pero también hay otras razones que están detrás de ésta elección: la adicción a drogas, el abuso sexual en la infancia o la violencia de género (Cobina y Oselin, 2011). Se ha planteado que en momentos de crisis económica aquellas actividades más informales, marginales o precarias, por las condiciones que conllevan su realización, aumentan considerablemente. Este puede ser el caso de algunas modalidades del mercado servicios sexuales, ya que la prostitución puede realizarse de forma permanente, ocasional o de manera puntual, dependiendo de las circunstancias de las personas que las efectúan. En momentos donde la tasa de paro nacional supera el 26\% en España (INE, 2013), con pocas ofertas de empleo, la prostitución resulta una alternativa, o un recurso complementario, a los escasos ingresos que se puedan obtener desde actividades formales para satisfacer las necesidades básicas. De hecho, en épocas de mayor bonanza la prostitución ha supuesto no solo una forma de subsistencia sino una buena opción para obtener importantes beneficios en los proyectos personales.

Pero también se ha realizado una distinción entre las mujeres que realizan estos intercambios de forma voluntaria de aquellas que son explotadas o coaccionadas para ejercer la prostitución. En estos casos las condiciones son muy diferentes y cambia radicalmente las ventajas que la actividad pueda tener para las mujeres. La distinción de estas dos realidades en muchas ocasiones no es fácil, pues la amenaza y el maltrato impiden que se reconozcan y se declaren las situaciones de prostitución coactiva, aunque en muchas ocasiones se pueda producir posteriormente un tránsito al ejercicio de la prostitución voluntaria.

Encontramos claras diferencias de género en la ocupación de esta actividad. Mayoritariamente la oferta de servicios sexuales se concentra en las mujeres mientras que la demanda de estos servicios se produce por parte de 
los varones en la mayoría de los casos. Se ha planteado que las actividades económicas precarias, estigmatizadas, infravaloradas o poco reconocidas socialmente suelen ser ocupadas en mayor medida por las mujeres que por los varones (Vanwessenbeeck, 2001).

Podemos apreciar diversas formas de ejercer la prostitución, o maneras de contactar con los clientes, destacando tres modalidades (Meneses, 2008): la que contacta en la calle, la que se produce en los clubs de alterne, ya sean urbanos o de carretera, y la oferta de servicios sexuales en los pisos o casas de cita. Podríamos añadir una cuarta forma de contacto, aquella que surge mediante internet, pero en muchas ocasiones las modalidades anteriores se anuncian por esta vía, además de por la prensa escrita. La prostitución callejera suele ser la más minoritaria, en torno al $5 \%$, y la que más se ha descrito por los riesgos que conlleva, principalmente de agresión o violencia (Raphael y Shapiro, 2004). Sin embargo, se trata de la modalidad que menos comisiones tiene, es decir, las ganancias obtenidas por los servicios sexuales son en exclusividad para las personas que ejercen la prostitución. Aunque en la última década habría que añadir el riesgo punitivo, o a ser multada por utilizar la vía pública y, en este sentido, a compartir sus ganancias con los ayuntamientos correspondientes. En el nuevo siglo han proliferado las ordenanzas municipales prohibiendo la prostitución de calle con importantes repercusiones para las mujeres (Villacampa y Torres, 2013). La prostitución de calle es la más visible y por tanto la más estigmatizada y discriminada.

El objetivo de este estudio es describir los cambios y repercusiones que se han producido en las personas que ejercen la prostitución en la calle, en su contexto más inmediato y en las entidades que les presta algún tipo de apoyo como resultado de la reducción o eliminación de servicios sociales y sanitarios en Madrid. Estas medidas se enmarcan en políticas sociales aplicadas en la Comunidad de Madrid por la crisis económica acontecida entre los años 2011 y 2013 ante la demanda de reducir el déficit público del Estado.

\section{Material y métodos}

La investigación presentada es descriptiva, exploratoria y cualitativa. Se utilizaron dos técnicas de investigación: la observación participante (OP) y la entrevista abierta. La primera técnica se realizó en una zona tradicional de prostitución nocturna de calle, de cierto nivel adquisitivo ${ }^{4}$. En dicha zona existían

4. Comprende un par de manzanas entre las calles Capitán Haya y la Castellana. Se trata de una zona tradicional de alterne tanto en clubs, locales y apartamentos. Es una de las zonas de negocios de Madrid, a pocos metros de la torre Picasso. 
cuatro o cinco establecimientos de alterne que facilitaba la afluencia de clientes de servicios sexuales a la zona. La presencia de las personas que ejercían la prostitución comenzaba sobre las 11:30 hasta las 5:00 de la madrugada. Se realizó entre una y dos horas a la semana de observación, desde septiembre del 2012 a junio del 2013, suponiendo un total de unas 75 horas de observación aproximadamente. Las dos autoras de este trabajo llevaron un diario de campo de forma separada que permitía, posteriormente, triangular la información, suponiendo un elemento de rigor y calidad en la investigación cualitativa (Whittemore y otros, 2001; Pyett, 2003). Se trataba de una zona en declive a nivel de oferta de prostitución callejera, puesto que las autoridades locales y los propios vecinos de la zona habían intentado eliminar la presencia de esta prostitución nocturna. A través de la observación realizada, y desde las entrevistas conseguidas con las personas que desarrollaban esta actividad en la zona, calculamos una presencia de alrededor de 40 personas $^{5}$ que frecuentaban la calle ejerciendo esta actividad, aunque a distintas horas y en distintos días. La saturación de contenidos nos permitió dar por finalizada la $\mathrm{OP}$ en aquellos aspectos que eran fundamentales para cumplir con los objetivos de investigación planteados.

La segunda técnica de investigación utilizada fue la entrevista abierta, aplicada con dos tipos de sujetos-participantes: las mujeres que ejercían la prostitución, contactadas en la calle desde la técnica anterior; y los/as coordinadores/as o responsables de instituciones que estaban trabajando con estas personas en Madrid en ese momento. Las entrevistas llevadas a cabo con las mujeres fueron cortas, por término general, aproximadamente 15 o 20 minutos cada vez que acudíamos a la zona, planteando la misma pregunta a todas las que ese día estaban ejerciendo la prostitución. A todas las mujeres se les informó cuál era nuestro objetivo en la zona, la voluntariedad de responder a nuestras preguntas y nuestro compromiso de mantener la confidencialidad, tal y como se ha planteado para esta técnica (Kawulich, 2005). En solo dos casos se pudo efectuar una entrevista en mayor profundidad, en torno a una hora. Respecto a las entrevistas realizadas a las instituciones que trabajaban con personas que ejercían la prostitución, se planteó inicialmente llevarlas a cabo en ocho instituciones, pero solo se consiguieron cinco entrevistas en cinco organizaciones ${ }^{6}$. Tres entrevistas no se acometieron debido a diferentes causas (falta de tiempo de las entidades, negación a realizarla...). La duración

5. De estas personas cuatro mujeres eran ya conocidas por una de las autores al realizar en esta misma zona trabajo etnográfico en la década de los noventa (1993 a 1995).

6. Nuestro profundo agradecimiento a APRAMP, Cáritas, Fundación Cruz Blanca, Fundación Triángulo, y Colectivo Hetaira por facilitarlos la entrevista y la información requerida para esta investigación. 
Tabla 1. Guión de entrevista semiestructurada

\section{Guión entrevistas a entidades}

1. características de las personas atendidas, diferenciando en mujeres, varones y transexuales;

2. cuántas provienen de Trata; denuncias que se establecen; si la entidad asume la denuncia y lleva los casos.

3. Organización de la actividad y maneras y modos de intervenir. Recursos que proporciona a las personas que ejercen la prostitución

4. Acceso de los usuarios y usuarias a los recursos ofertados, cómo los perciben, necesidades no cubiertas y cubiertas.

5. Coordinación con otras entidades

6. Posicionamiento ideológico en la prostitución

7. Apoyos institucionales, administración, colectivos, asociaciones, etc.

8. ¿Qué recursos harían falta? ¿Por qué? ¿Mecanismos puestos en marcha para tratar de conseguirlos?

9. Impacto de la crisis en las personas que ejercen la prostitución y en la entidad.

Grafico 1. Categorías analíticas utilizadas

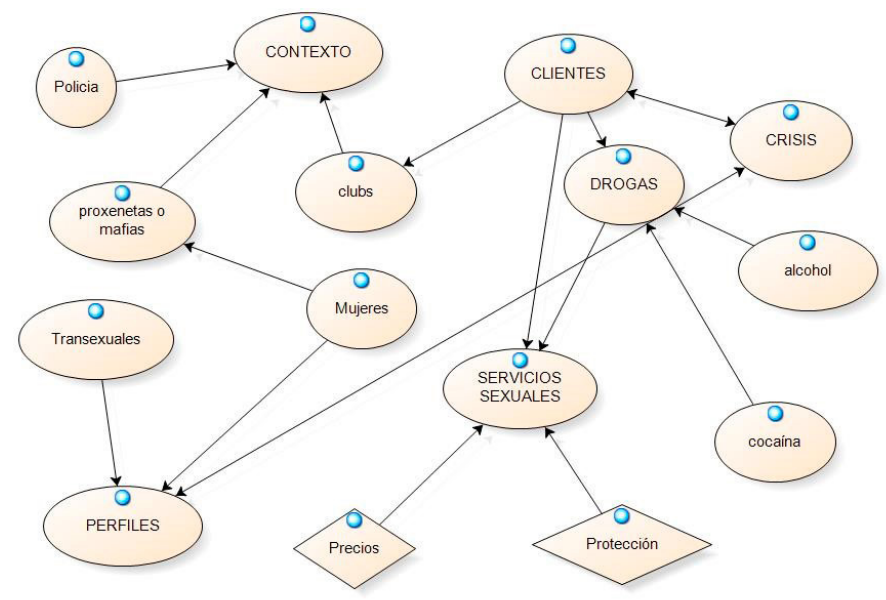

de las mismas fue entre 30 a 45 minutos y se efectuaron en la sede de las entidades. En la tabla 1 se presenta el guión de entrevista realizada. A pesar de que fueron cinco entrevistas, se consiguió la saturación de contenidos en las respuestas obtenidas al objetivo principal.

Se abordó un análisis categórico interpretativo de las notas de los diarios de campo y de las entrevistas realizadas. Se utilizaron distintas estrategias analíticas para los datos cualitativos (Leech y Onwuegbuzie, 2007): análisis de comparación constante; palabras clave usadas por los informantes en el contexto de estudio; análisis de contenido con categorías de análisis y codificación de los discursos y las notas; análisis de dominio o de los significados culturales. En el gráfico 1, se muestra las categorías analíticas 
utilizadas. Se utilizó el programa informático NVIVO v.10, en los procedimientos analíticos.

\section{Consideraciones éticas}

Todos los participantes fueron informados del objetivo del estudio, la participación fue voluntaria y se garantizó el mantenimiento del anonimato en los discursos y contenido obtenidos.

\section{Resultados}

Los datos recogidos nos llevan a señalar que las medidas de reducción de costes en servicios básicos como fruto de la crisis económica en los años mencionados afectaron a las personas que ejercían la prostitución, así como a las organizaciones que trabajaban con ellas. Mostraremos este impacto de forma separada, en las protagonistas y en las instituciones, pues los efectos fueron diferentes.

Impacto de la crisis en las personas que ejercen la prostitución

La gran mayoría de las mujeres contactadas en el trabajo etnográfico estaba ejerciendo la prostitución como medio de subsistencia. No obstante, para algunas de las mujeres jóvenes de Europa del este la entrada en la prostitución fue como consecuencia de una situación de coacción, engaño y explotación sexual, siendo menores de edad cuando sucedió esto. Este fue el caso de una de nuestras entrevistadas, con la que establecimos mayor relación. Una vez superada esta fase se había instalado en esta actividad como un medio de vida, y su juventud le proporcionaba importantes beneficios, siendo una de las pocas mujeres que la prostitución le proporcionaba en ese momento unos ingresos constantes y suficientes para sus necesidades. Pero no todas las mujeres que ejercían la prostitución en la calle conseguían mantenerse con esta actividad, ya que dependía de una serie de variables: si era autóctona o inmigrante, o la edad que tuviese. La juventud y la imagen física, o un cuerpo escultural, eran requisitos imprescindibles para atraer a los clientes y, por tanto, para disponer de un número diario de servicios y precios elevados en los mismos. En la zona estudiada la mayoría de las mujeres era joven y de Europa del este, especialmente de Rumanía. Las mujeres mayores, entre 38 a 50 años, conformaban un grupo destacado de españolas y extranjeras (brasileñas, italianas, colombianas, etc.). Muchas de estas mujeres habían disfrutado de prestaciones sociales o ayudas para ellas o sus hijos, complementando los ingresos de la prostitución, pero en ese momento se las habían suprimido 
(becas de comedor, becas para libros...). Por tanto, las percepciones sobre las consecuencias de la eliminación de servicios eran diferentes para cada una de ellas, porque partían de situaciones vitales distintas. En todo el tiempo que se realizó el trabajo etnográfico se recogió continuamente la queja sobre la escasez de clientes, si lo comparaban con otros momentos anteriores a este periodo. Los clientes eran su única fuente de ingresos.

«Ana está junto a la marquesina informativa del Ayuntamiento, junto a la acera. Se la nota muy enfadada. Parece que no hay clientes, según nos dice, lleva varios días aguantando largas horas y se marcha sin hacerse nada.» (Diario de campo, 13 diciembre, 2012)

«Nadine nos vuelve a comentar, como siempre, que no hay clientes, que ha estado bajando durante todo el mes de febrero y que no se ha hecho nada, que hay muy pocos clientes y que los que vienen no tienen dinero» (Diario de campo, 7 marzo 2013)

Se había producido una reducción de demanda de servicios sexuales, y esta era una consecuencia que ellas atribuían a la crisis económica. Las mujeres se acoplaban a las horas y días que encontraban mayor afluencia de clientes, alejados del fin de semana, o vísperas de fiestas. Por otra parte, al encontrarse la zona de prostitución muy cerca de un conocido estadio de fútbol, los días de partido entre semana eran días de gran ocupación. Esta zona de prostitución de calle, como sucede en otros lugares, estaba segmentada por edad. Las calles más visibles, transitadas, con afluencia de coches, y cercano a los dos grandes y prestigiosos hoteles, estaban reservadas para las chicas más jóvenes, fundamentalmente rumanas que eran las de mayor número. Las calles más oscuras y con menos tránsito eran el espacio de contacto de las mujeres más mayores. Dicha organización se mantenía desde la década de los ochenta y noventa, cuya presencia de mujeres en la zona cada noche fue más elevada que en ese momento.

La prostitución no conseguía ser la única fuente de ingresos para muchas de las mujeres, especialmente las más mayores, ya que la baja frecuencia de clientes demandando servicios sexuales les impedía que se mantuviera como su único medio de vida. Ello llevaba a que muchas de ellas articularan distintas estrategias para aumentar los servicios sexuales.

«Ana: Le he dicho al del bar qué cuanto me paga si me hago tarjetera, y me ha dicho que ya hablaremos. Claro, el tiempo que estoy aquí, esperando clientes, pues me dedico a repartir tarjetas del bar y me llevo unas pelas, que esto está mu, mal» (Entrevista, Ana, 40 años, española, 11 abril 2013)

Una manera de captación de clientes surgía aprovechando las nuevas tecnologías, dándose a conocer en páginas web de internet. Se buscaba maneras 
alternativas de captar clientes que permitiera aumentar los ingresos y poder satisfacer sus necesidades básicas.

«Nos habla de una colega rubia, que estaba con ella cuando nos acercamos a saludarla y que se ha marchado porque la han llamado por teléfono. Se anuncia en Internet, en una página web específica. Pero nos dice que ella no puede, que tiene un hijo de 21 años que no sabe a qué se dedica. Nos comenta que en las páginas web aunque pongas la cara de refilón suele haber fotografías del cuerpo, para que los clientes te vean y seleccionen, y que ella no puede porque tiene varios tatuajes que sería reconocidos rápidamente por su hijo, sus amigos o sus familiares.» (Diario de campo, 14 marzo, 2013)

Otra manera era el trasiego de las mujeres hacia el club y desde éste hacia la calle, aspecto que ha sido comentado en otras ocasiones y estudios (Day, 2007) y que se agudizaba en ese momento. La trayectoria solía ser del club a la calle, pues los ingresos que las mujeres obtenían, si no había proxeneta o mafia detrás que controlasen sus recursos, eran exclusivamente para ellas. Sus discursos también señalaban una baja demanda de servicios sexuales en los clubs de alterne, cuando venían de estos locales a ocupar espacios en la calle. Así nos lo contaba una de las mujeres.

« $i$ Te has planteado trabajar en un club?

No, porque tienes que ir al $50 \%$ con el dueño, en copas y en todo. No me interesa.» (Diario de campo, 8 noviembre 2012)

Era posible que la salida de club estuviera motivada por diferentes circunstancias, como el consumo de drogas, la mayor edad, etc. La edad era muy importante. Las mujeres mayores, a partir de los 40 años aproximadamente, difícilmente eran admitidas en los clubs o locales de alterne de la zona, ya que eran considerados como alto standing y eso significaba disponer de mujeres con muy buena presencia. Existía alguna excepción en ciertos pisos o apartamentos $^{7}$, tolerándose la presencia de mujeres de mediana edad junto con las mujeres más jóvenes.

«Esta noche conocemos a Nadia, es colombiana, pero lleva residiendo más de 20 años en España. Ha trabajado siempre en un club de alto standing de Madrid, pero ahora está en la calle. Aunque ella justifica su presencia en la calle de otra forma, el hecho es que a pesar de tener una apariencia e imagen estupenda, tiene 50 años, aunque aparente diez años menos. Si siguiese en el

7. Alrededor de la zona de prostitución de calle se encuentran una serie de pisos o apartamentos que son previamente concertados con las mujeres que están en la calle para poder realizar los servicios. También los tres grandes hoteles son otra opción, principalmente si son clientes de estos alojamientos. Por último, también es una zona de muchos apartamentos destinados a la venta de servicios sexuales. 
club la demanda de clientes hacia ella sería muy escasa» (Diario de campo, 17 septiembre 2012)

Solo se justificaba salir de la calle a un club, desde sus propias perspectivas en estos momentos de crisis económica y escasez de recursos, por dos motivos: las agresiones o violencia a la que se exponían en la calle o por las condiciones meteorológicas. Fue el caso de Catalina, una joven rusa, que se marchó de la zona porque había sido sujeto de agresión por parte de un cliente que iba bebido.

Por último, donde más afectaba la situación de crisis era a los servicios sexuales, especialmente en el precio y en el tipo de servicio. El sexo oral era la practica más demandada a las mujeres que contactaban en la calle, ya que dicho servicio era rápido y el más económico, pudiéndose realizar en el mismo coche del cliente. Un sector de los clientes que compraban estos servicios sexuales los solicitaba sin protección y se recorría la zona comparando precios y buscando la persona que se los ofreciese sin preservativo.

«Cuando llegamos Ana está negociando en un coche. Esperamos un momento por si no se lleva a efecto la negociación. Efectivamente se marcha. Nos acercamos a ella y nos dice que el cliente le pedía sexo oral sin preservativo. Hoy ha venido pronto, sobre las 22:00 y son 00:40, a.m y no se ha ocupado con nadie... Vemos a Nadia, se queja de lo mismo que Ana, ningún cliente y el único que se le ha acercado quería sexo oral sin preservativo. Parece que es el mismo, está buscando quién le oferta lo que quiere. Además, quiere el servicio por 20 euros». (Diario de campo, 21 febrero, 2013)

No hemos podido constatar que alguna de las mujeres ofertase los servicios sin protección, pero referencias a que siempre había alguna de ellas que rompía las normas y ejercía una competencia desleal estaba en sus discursos. Especialmente llamativo resultaba dicha acusación de las españolas y de mayor edad hacía las mujeres extranjeras, -rumana o albanesa-. Una de nuestras informantes de la calle nos decía:

«Mira, yo cuando me piden los servicios sin preservativo, les digo que son 500 euros, para quitármelo de encima. Una vez le dije a uno que cuando me trajera los análisis y me mostrara que no tenía ninguna enfermedad. Y el cliente vino otro día con las analíticas. Nos estuvimos riendo un rato de él». (Entrevista Adriana, 23 años. Rumana).

Por último, otro de los servicios demandados fue el consumo de drogas con el cliente, especialmente cocaína. En este caso no parecía que el precio hubiera variado, aunque la demanda del mismo sí.

«Tengo un cliente fijo, que es profesor de la Universidad como tú, que se pone coca y quiere que consuma con él. Tengo muchos clientes que consumen coca, o te viene puestos de coca, o te quieren pagar en coca porque no tengan 
pelas pero tengan coca, pero yo nunca lo acepto». (Entrevista Adriana, 23 años, Rumana).

En la zona estudiada tenían presencia puntual dos organizaciones, que antiguamente prestaban los servicios todas las semanas. Sin embargo, solo coincidimos con una de ellas y solo un día. La reducción de subvenciones por parte de la administración pública madrileña, como veremos a continuación, hizo que estas organizaciones tuviesen que disminuir su presencia en esta zona de prostitución. Por otra parte, durante gran parte del tiempo del trabajo de campo realizado se observaron controles policiales, a veces de alcoholemia y otras de cualquier tipo, que mermaban la presencia de mujeres y de clientes. Como ya hemos mencionado, la zona disponía de varios clubs de alterne que generaba una afluencia de clientes potenciales por la calle y que hacía muy difícil erradicar totalmente la prostitución en la vía pública.

Impacto de la crisis económica en las Organizaciones de apoyo

Las cinco entidades entrevistadas tenían presencia en las principales zonas de prostitución del Municipio de Madrid, y todas ellas confirmaban la información que habíamos recogido previamente: se había producido una disminución de la demanda de servicios sexuales y las mujeres que ejercían la prostitución no lograban obtener los recursos económicos que necesitaban para vivir.

«La crisis ha afectado en gran medida al mundo de la prostitución. Hay menos actividad, menos clientes... las mujeres que viven en el propio club donde ejercen la prostitución, vale... pero las que viven por su cuenta tienen serias dificultades para sufragar los gastos» (ONG, religiosa)

Por tanto, las entidades entrevistadas triangulaban la información que había sido recogida en el trabajo de campo etnográfico, y podía ser extrapolada a todas las zonas y sectores donde trabajaban. Esta escasez de clientes y de ingresos estaba generando una serie de consecuencias y repercusiones para las mujeres:

En primer lugar, si la prostitución para un sector de mujeres era una actividad temporal, hasta que la crisis económica remitiera, se estaba convirtiendo en algo más prolongado y no previsto inicialmente, además de pasar más horas intentando contactar con los clientes.

«Anteriormente las mujeres que venían a España a ejercer la prostitución lo consideraban como una «etapa provisional» o "proyecto de vida», como un medio para poder tener suficientes ingresos y luego emprender una vida independiente. Estos proyectos vitales se han visto destrozados, sus 
posibilidades son muy restringidas...dejar esto es prácticamente imposible» (ONG Religiosa)

«Las personas prostituidas trabajan muchas más horas que antes» (ONG, Religiosa)

En segundo lugar, la disminución de clientes, o de demanda de servicios sexuales, se producía en un momento de aumento de la oferta de estos servicios; es decir, más mujeres recurrían a la prostitución para sobrevivir. Incluso era el caso de muchas mujeres que hacía tiempo habían abandonado la prostitución, y entre las que predominan españolas. Por tanto, no solo había en la zona mujeres extranjeras.

«...de alguna manera tienen que satisfacer sus necesidades básicas...y en la prostitución tienen experiencia y contactos» (ONG Religiosa)

«Antes la mayoría provenían de Brasil, el Magreb, Europa del Este o Latinoamérica. Ahora muchos son españoles. Se nota en este sector también lo mucho que está sufriendo la población española en relación al empleo». (ONG laica, desde 1996)

En tercer lugar, ese aumento de oferta, con una disminución de la demanda, conducía al aumento de la competitividad entre ellas y a la reducción de la solidaridad o ayuda mutua.

«Esto supone mayores tensiones y conflictos entre ellas, y una reducción de la red de apoyo inmediata que allí tienen». (ONG, Laica, desde 1995)

En cuarto lugar, la escasez de ingresos había influido en la aceptación de algunas prácticas de riesgo. Solo se podía asumir una desprotección, o no usar preservativos, en los servicios sexuales si el precio de los mismos se triplicaba.

«Algunas se someten incluso a prácticas de alto riesgo, y antes nunca lo harían» (ONG laica, desde 2011)

En quinto lugar, otro servicio que podía incrementar los ingresos era el consumo de drogas. Los clientes, que acudían a la zona y las consumían, deseaban que las personas con las que se ocupaban y a las que le demandaban servicios sexuales las consumieran con ellos. Habitualmente las mujeres no ofrecían este tipo de servicio, pero muchos de ellas comenzaron a admitirlo.

Las drogas que se consumen ahora son principalmente cocaína y alcohol. Lo hacen para aguantar más. Un día se dan cuenta de que su compañera aguanta más que ellas, y lo prueban. Empiezan queriendo aguantar más y acaban volviéndose adictas. A veces son ellas mismas las que compran cocaína, pero otras muchas son los propios hombres quien se las facilitan. (ONG laica, desde 2011).

El sexto lugar, todas las entidades habían sufrido recortes en las subvenciones que recibían de la administración y que les permitían realizar su trabajo 
con las mujeres en los contextos de prostitución. Esta merma de ingresos había supuesto la reducción de su presencia en los contextos de calle y de su intervención.

Hemos perdido muchas subvenciones de nuestros proyectos de prostitución, si ya de por si es un sector muy excluido ahora lo está más. Los proyectos de prostitución sobreviven fundamentalmente por los fondos propios que aporta XXX por propia convicción, por aportaciones particulares, y por el apoyo tanto humano como material de las Adoratrices (Proyecto Esperanza) y las Oblatas. (ONG, religiosa)

Por último, una crítica importante recogida de todas las organizaciones fue las reducciones en materia de salud. La normativa aplicada en la Comunidad de Madrid en esos años impedía que los inmigrantes no regularizados pudieran ser atendidos en los servicios sanitarios, salvo en caso de urgencia. Esto suponía que la gran mayoría de las mujeres que ejercía la prostitución en esta zona no tenía acceso a los servicios sanitarios, pues estas mujeres eran extranjeras y en situación irregular. Por último, el material sanitario ofrecido a estas personas, especialmente los preservativos, también se había reducido e incluso mermado en calidad.

Esto es un gran problema, en el sexo anal es necesario tanto preservativo como lubricante, si no se rompe, y se contagia el Sida. Ahora ya no recibimos ni lubricante, y no podemos hacernos casi cargo de ese gasto...Es un problema de salud pública, un problema muy serio. (ONG, laica desde 1996)

Es muy importante el acceso a la atención sanitaria, y no solo para atender la salud sexual, sino porque el personal sanitario hacía una importante labor de detección de casos de $\operatorname{Trata}^{8}$, prostitución o malos tratos». «Esto las deja todavía más aisladas, todavía más fuera de la red normalizada de recursos. (ONG, religiosa).

Las instituciones públicas delegaban en las ONGs ciertos servicios sociales y de información sanitaria para las personas que ejercían la prostitución. Si estas organizaciones no podían seguir cumpliendo esta tarea como venían haciendo hasta el surgimiento de los recortes económicos el resultado era la

8. Trata hace referencia a la explotación de la prostitución forzada, más en concreto nos referimos a casos de Trata cuando se produce «la captación, el transporte, el traslado, la acogida o la recepción de personas, recurriendo a la amenaza o al uso de la fuerza u otras formas de coacción, al rapto, al fraude, al engaño, al abuso de poder o de una situación de vulnerabilidad o a la concesión o recepción de pagos o beneficios para obtener el consentimiento de una persona que tenga autoridad sobre otra, con fines de explotación. Esa explotación incluirá, como mínimo, la explotación de la prostitución ajena $\mathrm{u}$ otras formas de explotación sexual, los trabajos o servicios forzados, la esclavitud o las prácticas análogas a la esclavitud, la servidumbre o la extracción de órganos»(ONU, 2004:44-45).La Trata puede ser con fines de explotación sexual, laboral o de órganos. 
desatención básica a colectivos sociales vulnerables, incrementando la exclusión social.

\section{Discusión}

Como en otros estudios (Meneses et al, 2003; Cobbina y Oselin, 2011), las personas que ejercían la prostitución en la calle presentaban distintas trayectorias de entrada en la actividad, que se enmarcaban en los dos tipos de prostitución: la prostitución voluntaria y la prostitución coactiva. En ambas la crisis económica tenía importantes repercusiones para su bienestar social y su salud, afectado negativamente en estos dos aspectos a los colectivos más vulnerables. Nuestros resultados son acordes con otros trabajos donde se señalaba que la principal motivación para ejercer la prostitución en el colectivo de calle era la necesidad económica (McKeganey y Barnard, 1996). De hecho, algunas mujeres que habían dejado la prostitución recurrían de nuevo a ella para poder sobrevivir, especialmente las autóctonas y las más mayores de la zona (más de 40 años).

Además de la crisis económica, las medidas represivas, que pretendían erradicar o invisibilizar a ciertos colectivos sociales de la vía pública, generaban consecuencias más negativas que positivas, principalmente en materia de salud pública. En este sentido, tanto los escasos recursos económicos como los excesivos controles y sanciones administrativas podían producir una mala negociación de los servicios sexuales entre las personas que ejercían la prostitución y sus clientes, con importantes repercusiones en la protección contra las infecciones de transmisión sexual. Los servicios sexuales sin preservativos han sido demandados siempre por cierto sector de clientes y aunque la gran mayoría de las mujeres lo ha rechazado, en situaciones de crisis económica pueden considerarse como una opción para obtener ingresos (Cusick, 1998).

Nuestros resultados concuerdan con lo planteado por Adriaenssens y Hendrickx (2012), en donde el segmento de mercado de la prostitución más bajo, la de calle, la mayor edad de las personas que ejercían la prostitución y el menor atractivo físico estaban detrás de la aceptación de prácticas sexuales sin protección. El incremento del riesgo -vivido como algo lejano- era compensado por la obtención de ingresos económicos que les permitía subsistir a corto plazo.

También nuestros resultados apuntan a lo encontrado en otros trabajos (McKeganey y Barnard, 1996, Gossop et al, 1994, Meneses, 2007b y 2010), esto es, las conexiones entre el consumo de drogas y el consumo de servicios sexuales por parte principalmente de los clientes y secundariamente de las personas que prestan estos servicios, con las consecuencias que generan para 
la salud y el bienestar. Existen pocos y escasos programas de prevención del consumo de drogas en los contextos de prostitución, y los riesgos de adicción a cocaína y alcohol, como sustancias principales de consumo en estos contextos, pueden producirse en ambos actores sociales.

En ese momento la gran mayoría de las prestaciones sociales (alimentos, pago de libros, ropa, becas de guardería, comedor, etc.) que recibía la población que se dedicaba a la prostitución de calle eran ofrecidas por las organizaciones no gubernamentales, no existiendo recursos públicos para este sector. Si estas organizaciones que vienen desarrollando una tarea de atención y prevención a estos colectivos, desde los recursos que recibían del Estado, dejan de hacerlo queda al descubierto la atención social a un colectivo vulnerable y necesitado.

Las repercusiones de la inaccesibilidad a los servicios sanitarios por parte de las personas inmigrantes que ejercen la prostitución puede generar secuelas importantes tanto en esta población como en el trabajo que realizan las ONGs. En primer lugar, porque para muchas mujeres que ejercían la prostitución el mantenimiento del contacto con estas organizaciones se realizaba por la información sanitaria y el reparto de preservativos que les ofrecían, siendo una herramienta fundamental de trabajo. Desde esta tarea las organizaciones podían detectar aquellas mujeres que estaban explotadas o eran víctimas de Trata, y esta tarea se ponía en peligro. En segundo lugar, siendo un colectivo que necesitaba cuidar su salud se estaba poniendo en riesgo no solo su salud sino la de su familia y el resto de la población. Aunque fuera de forma egoísta debería replantearse que la falta de atención a los colectivos inmigrantes repercute en la falta de control de enfermedades e infecciones para el resto de la población. La atención sanitaria básica es una cuestión de derechos humanos y derechos de ciudadanía y España, como otros países de la Unión Europea, debería ser modelo en esta materia, ya que ha firmado y se ha vinculado a los tratados internacionales en materia de derechos humanos y de erradicación de la Trata, como el Protocolo de Palermo (2000),

Algunas limitaciones hacen que no podamos generalizar los resultados obtenidos, ya que no han sido explorados todos los contextos de prostitución de calle en Madrid, sino solo uno de ellos. Por otra parte, la presencia de varones y transexuales fue muy reducida, lo que imposibilita también extender los resultados a estos colectivos.

De los resultados obtenidos podríamos concluir con dos recomendaciones tanto para la intervención como para la investigación social. En primer lugar, evitar la reducción de recursos económicos en las entidades que trabajan con las personas que ejercen la prostitución en la calle, pues repercute en 
la oferta de atención y de servicios que pueden ofrecer, a la vez que se reduce considerablemente su labor de detección, información y prevención en los aspectos socio-sanitarios. En muchos pisos, locales de alterne e incluso carreteras o vías públicas donde se ejerce la prostitución el único contacto que tienen estas personas fuera del contexto de la prostitución son los profesionales que trabajan en las ONGs. La ausencia de las entidades de estos contextos de prostitución puede impedir la detección de la prostitución coactiva, y más en concreto la Trata con fines de explotación sexual en mujeres y niñas, fenómeno que ha ido creciendo en España en los últimos años (EUROTAT, 2013). En segundo lugar, se debe facilitar el acceso de estos colectivos vulnerables a la salud universal, pues el que solo puedan ser atendidos desde urgencias hospitalarias supone un gran desconocimiento de la epidemiología y la salud pública por parte de los políticos madrileños (como de hecho posteriormente se ha revocado). Estas medidas impiden tratar a tiempo enfermedades infecciosas y disminuyen el bienestar y calidad de vida de las personas, algo que atenta contra los derechos humanos y resulta increíble en sociedades democráticas y solidarias como las europeas.

\section{Bibliografía}

AdriaensSens, S., y Hendrickx, J. (2012).Sex, Price and preferences: accounting for unsafe sexual practices in prostitution markets. Sociology of health \& Illness, 34(5), 665-680.

CoBbiNA, J.E., y OSELIN S.S. (2011). It's Not only for the money: an analysis of adolescent versus adult entry into Street prostitution. Sociological Inquiry, 82(3), 310-332.

Cusick, L. (1998). Female Prostitution in Glasgow: Drug Use and Occupational Sector. Addiction Research, 6(2), 15-30.

FLICK, U. (2004). Introducción a la investigación cualitativa. Madrid: Morata.

Gossop, M. Powis, M. GrifFithS, P. y StranG, J. (1994) Sexual Behaviour and Its Relationship to Drug-Taking among Prostitutes in South London. Addiction, 89(8), 961-70.

KAWULICH, B.B. (2005). La observación participante como método de recolección de datos. Qualitative Social Research, 6(2),1-32

LeECH, N.L., y OnwUeGbuZIE, A.J. (2007). An Array of Qualitative Data Analysis Tools:bA Call for Data Analysis Triangulation. School Psychology Quarterly, 22(4), 557-584.

McKeganey y Barnard, (1996). Sex Work on The Streets. Prostitutes and Their Clients. Buckingham: Open University Press. 
Meneses, C., Rubio, E., Labrador, J., Huesca, A., y Charro, B. (2003).Perfil de la prostitución callejera. Análisis de una muestra de personas atendidas por APRAMP. Madrid: Universidad P. Comillas.

MENESES, C. (2007). Riesgo, vulnerabilidad y prostitución. Documentación Social, 144, 11-35.

MENESES, C. (2007b). Consecuencias del uso de cocaína en las personas que ejercen la prostitución. Gaceta Sanitaria, 21(3), 191-196.

MenEses C. (2008). Prostitución y salud mental, en Achotegui J. (Ed). Exclusión social y salud mental. Curso acreditado de formación continua para atención primaria. Barcelona, Ediciones Mayo. Pp.82-90.

Meneses, C. (2010). Usos y abusos de drogas en contextos de prostitución. Revista Española de Drogodependencias, 35(3), 329-344.

Monroe, J. (2005). Women in Street Prostitution: The Result of Poverty and the Brunt of Inequity. Journal of Poverty, 9(3), 69-88.

ONU. (2004). Convención de las Naciones Unidas contra la delincuencia organizada transnacional y sus protocolos. 44-45. http://www.unodc.org/documents/treaties/UNTOC/Publications/TOC\%20Convention/TOCebook-s.pdf (Consulta, 11 nov 2015)

PyetT, P. (2003). Validation of Qualitative Research in the «Real Word». Qualitative Health Research, 13(8), 1170-1179.

RAPHAEL, J. y SHAPIRO, D. (2004) Violence in Indoor and Outdoor Prostitution Venues.» Violence Against Women, 10(2), 126-39.

Whittemore, R., Chase, S., y Mandle, CL. (2001). Validity in Qualitative Research. Qualitative Health Research, 11 (4), 522-537.

Willman, A. (2010). Let's talk about money. En M. Hope, A. Levy y A. Willman (Eds.). Sex Work Matters. London: Zed Books.

VANWESSENBEECK,I. (2001). Another Decade of Social Scientific Work on Sex Work: a review of research 1990-2000. Annu Rev Sex Res, 12:242-89.

VillacAmpa, C., Y TORRES, N. (2013). Políticas criminalizadoras de la prostitución en España. Revista Electrónica de Ciencia Penal y Criminología. 15-06:1-40. 